\title{
Efectos de la suplementación de hierro sobre el desarrollo mental y psicomotor de los niños
}

\author{
Effects of iron supplementation on mental and psychomotor development of children
}

Szajewska H y col. Am J Clin Nutr. 2010 Jun;91(6):1684-90.

\section{Objetivos}

Evaluar los efectos de la suplementación con hierro en mujeres embarazadas y/o niños menores de tres años sin anemia en el ulterior desarrollo mental y psicomotor de dichos niños.

\section{Fuente y selección de datos}

Los autores seleccionaron ensayos clínicos aleatorizados y controlados con placebo o con un grupo de "no intervención" que hasta 2009 estuvieran indizados en MEDLINE, EMBASE y en la bibloteca Cochrane y que hubieran incluido alguna medición estandarizada del desarrollo psicomotor, la función cognitiva y/o la conducta de los niños.

\section{Resultados}

Luego de la exclusión de 40 ensayos clínicos iniciales fueron analizados siete ensayos.

Dos estudios habían incluido una población de embarazadas sin anemia $(n=430)$ de Australia, a cuyo grupo intervención se le había indicado la ingesta oral de $20 \mathrm{mg}$ de Hierro elemental desde la vigésima semana de embarazo y hasta el parto. Cinco estudios habían incluido niños menores de tres años, a quienes se les había prescripto una dosis que iba de 7,5 a $10 \mathrm{mg} /$ día, desde los tres meses y hasta los 15 meses de vida.

Para medir el desarrollo psicomotor se había utilizado la escala Bayley ${ }^{1}$ del Desarrollo Infantil (en inglés: Bayley Scales of Infant Development o BSID) que evalúa lenguaje, habilidades visuales y motoras, y comportamiento. De estas mediciones fueron derivados el Índice de desarrollo psicomotor (en inglés:
Psychomotor Developement Index) y el Índice de Desarrollo Mental (en inglés: Mental Development Index o MDI).

Ningún estudio mostró individualmente beneficios de la suplementación de hierro en el desarrollo mental de los niños en ningún punto del estudio (MDI y BSID) ni tampoco el metaanalisis de tres ensayos clínicos $(n=561)$.

Respecto del desarrollo psicomotor, tres de cinco estudios mostraron un beneficio de la suplementación en el PDI a diferentes puntos de medición. Un meta análisis, realizado sobre la base de tres de estos ensayos clínicos $(n=561)$ informó una mejoría significativa al año de edad en el grupo asignado a suplementación oral (diferencia media ponderada en el PDI de 4,21; IC95\%: 2,31 a 6,12).

Respecto de la suplementación de hierro durante el embarazo, no se hallaron beneficios significativos en relación al coeficiente intelectual y/o al comportamiento de los niños.

\section{Conclusión}

Solo algunos estudios documentaron que el suplemento con hierro durante el embarazo y/o en niños menores de tres años se asocia a una mejoría de su desarrollo psicomotor, sin que se pudieran observarar beneficios en el desarrollo mental.

Fuente de financiamiento: Commission of the European Communities, research project "The effect of diet on the mental performance of children".

\section{Comentario}

Consideramos que la variables que pueden valorarse a través de los instrumentos de medición utilizados en estos trabajos no son capaces de definir por sí mismas y en forma completa cuestiones tan complejas como inteligencia o desarrollo mental. Estas, sin duda están también relacionados con determinantes como la cultura, el ambiente, etc., y no sólo con los niveles de hierro.

Además, no existe consenso respecto de la relevancia clínica de una diferencia de 4,2 puntos en el puntaje PDI; destacándose también la problemática del sesgo de selección ya que los propios autores reconocen que seguramente existe un importante número de trabajos no publicados (particularmente los realizados en países subdesarrollados) que de ser identificados, quizás influenciarían los resultados globales de una revisión sistemática como esta.

\section{Conclusiones de los comentadores}

Es importante remarcar que estos resultados provenientes del metanálisis de Szajewska y col. que hemos resumido dependen de datos de ensayos clínicos individuales que habían sido llevados a cabo en países como Canadá y Australia, y en los que la prevalencia de anemia y quizás su impacto sea mucho menor que en la nuestra; por lo que consideramos que todavía no está zanjada la pregunta respecto de la evidencia que sustenta nuestra práctica de suplementar con hierro casi en forma rutinaria a nuestros pacientes provenientes de estratos socioeconómicos bajos.

Pedro Silberman y Silvina López [ Area Salud Individual y Colectiva, Rotacion Medicina Familiar, Carrera de Medicina, Departamento de Ciencias de la Salud, Universidad Nacional del Sur: pedrosil2005@yahoo.com.ar, lopezsil2001 @ hotmail.com ]

Silberman P y Lopez S. Efectos de la suplementación de hierro sobre el desarrollo mental y psicomotor de los niños. Evid Act Pract Ambul. Oct-Dic 2011, 14(4) 127. Comentado de: Szajewska H y col. Effects of iron supplementation in nonanemic pregnant women, infants, and young children on the mental performance and psychomotor development of children: a systematic review of randomized controlled trials. Am $\mathrm{J}$ Clin Nutr. 2010 Jun;91 (6):1684-90. PMID: 20410098.

\section{Referencias}

1. Bayley M. Bayley Scales of Infant and Toddler Development, 3er edition. BAYLEY-III, 2005. 